\title{
ANTIQUITÄT IN EINEM FRÜHHUMANISTISCHEN WERK. JOHANNES TRÖSTERS DIALOGUS DE REMEDIO AMORIS
}

\begin{abstract}
Summary: The paper reviews the antique models of Johannes Tröster's Dialogus de remedio amoris (1454). Obviously, the most important inspiration for this work was the Remedia amoris by Ovid but other antique writers, e.g. Seneca, Plautus, Terence or Plutarch also had a remarkable influence on this literary work. In particular, the allusion to Virgil's gates of sleep is noteworthy, which supports the reader's interpretation of the dialogue. Last but not least: efforts have been made to relate Tröster's own manuscripts of these models based on information about the contents of his library.
\end{abstract}

Key words: Johannes Tröster, Eneas Silvius Piccolomini (Pope Pius II), Johannes Vitéz de Zredna, Janus Pannonius, humanism in Hungary in the 15th century, Ovid, Plautus, Terence, Vergil, the gates of sleep, Seneca, Plutarch, Avicenna, Valerius Maximus

\section{TRÖSTER UND DER DIALOGUS DE REMEDIO AMORIS}

Commovet enim constantes animos relecta Sirenearum melodiarum pulsatio ${ }^{1}$ - Das kann im Allgemeinen für die Liebe gültig sein, aber das ist keinesfalls gesetzmäßig in der Rezeption eines literarischen Werkes. Die oben zitierte Ovid-Allusion ist im Dialog (verfasst im Jahre 1454) des Johannes Tröster (um 1425-1485), einem der zu Unrecht vergessenen Stücke der frühneuzeitlichen Literatur zu lesen: Das Werk ist, wie der Autor, der hoffnungsvolle Literat selbst, in Vergessenheit geraten. Jedoch begann seine Karriere erfreulich: Nach den Wiener Universitätsstudien wurde er als

* Hier möchte ich mich bei Frau Claudia Märtl, Herrn Franz Josef Worstbrock und Franz Fuchs für ihre freundliche Hilfe bedanken. Dieser Aufsatz wurde auf Ungarisch bereits in der Zeitschrift Ókor 11 (2012) 37-43. veröffentlicht.

${ }^{1}$ Auszug aus Trösters Dialog, vgl. Ovid. Rem. Am. 718: Constantes animos scripta relecta movent. Mangels einer kritischen Ausgabe muss die kontaminierte Textedition von Hans Rupprich zitiert werden. RUPPRICH, H.: Die Frühzeit des Humanismus und der Renaissance in Deutschland. Leipzig 1938, 183197, hier 191. 
Familiare von Eneas Silvius Piccolomini, dem späteren Papst Pius II. Erzieher solcher Prominenten, wie der spätere Papst Pius III. und der damalige König Ladislaus V. 1452 verlor er seine Stelle unter dem Verdacht der Beteiligung an Ladislaus' Fluchtversuchen und fiel in Ungnade. Zwei Jahre lang versuchte er die Gnade des Kaisers Friedrich III. wieder zu erhalten, was ihm aber erst um 1457 gelang. ${ }^{2}$ Seine Entbehrungen nahmen ein Ende als er dank der gewiss an Johannes Vitéz gerichteten Redaktion des Dialoges in seinen Dienst trat: Von 1454 bis 1457 war er mutmaßlich als Gesandter zwischen Vitéz, Piccolomini und dem Salzburger Bischof Sigismund I. von Volkersdorf tätig. In der v.a. bayerisch-österreichischen Forschung wird er vorwiegend als „humanistisch gesinnter Wohltäter bayerischer Büchersammlungen“3 benannt, in diesem Themenbereich sind schon zahlreiche Publikationen erschinen, da immer neuere Bände seiner ehemaligen Bibliothek zum Vorschein kommen. ${ }^{4}$

Der Titel des Werkes, Dialogus de remedio amoris (im Folgenden: Dialogus) verweist offensichtlich auf die ovidianischen Vorbilder, v.a. auf die Remedia amoris, während er auch den Brief seines Lehrers Eneas Silvius Piccolomini, der unter dem Titel De remedio amoris illiciti bekannt ist, hervorruft, manchmal auch zitiert: ${ }^{5}$ Tröster imitiert gleichzeitig die antiken Vorgänger und die zeitgenössischen Muster. In diesem Aufsatz werden einige Beispiele dargebracht, die veranschaulichen, wie Tröster auf die meist zitierten antiken Autoren zurückgreift - sie gelten auch als Zusätze zur Rezeptionsgeschichte der antiken Literatur. In der Mehrzahl der Fälle werden nur die Paraphrasen (mit den entsprechenden Kontexten) kurz angegeben, aber einige Passus werden einerseits wegen ihrer rezeptionsgeschichtlichen Relevanz, andererseits wegen ihrer herausragenden Rolle bei der Interpretation des Dialogus umfangreicher untersucht und kommentiert. Es wird außerdem versucht, die eventuellen literarischen Vorbilder, soweit möglich, den Stücken der rekonstruierten Bibliothek zuzuordnen.

\section{OVID}

Auf die Remedia amoris als primäres, prägendes Vorbild wird schon mit dem Titel verwiesen. Alle Stellen, an denen die Heilmittel gegen die Liebe behandelt werden, stammen aus diesem Werk.

\footnotetext{
${ }^{2}$ Tröster an Kaspar Wendel, s. Brno, UB, Mk 96, 155rv. Undatierter Brief, terminus post quem ist der 17. November 1457, da Tröster über den Tod des Königs Ladislaus schreibt.

${ }^{3}$ LEHMANN, P.: Dr. Johannes Tröster, ein humanistisch gesinnter Wohltäter bayerischer Büchersammlungen. Historisches Jahrbuch 60 (1940) 646-663.

${ }^{4}$ LeHMANN (Anm. 2); CORTESI, M.: Zur Büchersammlung des Johannes Tröster $(\dagger 1485)$. In FuCHS, F. (Hrsg.): Der Humanismus an der Universität Leipzig [Pirckheimer Jahrbuch 23, 2008]. Wiesbaden 2009, 217-231; SCHULLER-JUCKES, M.: Johannes Tröster als früher Auftraggeber des Salzburger Buchkünstlers Ulrich Schreier. In FUCHS 233-245.

${ }^{5}$ Die Ausgabe des Briefes von Piccolomini s. Wolkan, R. (Hrsg.): Der Briefwechsel des Eneas Silvius Piccolomini: Abt. 2. Briefe als Priester und als Bischof von Triest [Fontes rerum Austriacarum 67]. Wien 1912, 33-39. Trösters Imitation wurde von Georg Braungart behandelt, allerdings verwies er nicht auf die exakten Zitate, sondern verfasste nur einen thematischen Vergleich, s. BRAUNGART, G.: De remedio amoris: Ein Motiv und seine Traditionen von der Antike bis Enea Silvio Piccolomini und Johannes Tröster. Archiv für Kulturgeschichte 62/63 (1980/1981) 11-28.
} 
Qui non est hodie, cras minus aptus erit:

Verba dat omnis amor, reperitque alimenta morando

(...)

Vidi ego, quod fuerat primo sanabile, vulnus

Dilatum longae damna tulisse morae.

(Rem. Am. 94-95 und 101-102)

Nec cras, cras dicito, cum hodie facere queas: reperit enim in morando alimenta amor. Vidi namque sanabile vulnus in mortiferum virus longam redigere moram. ${ }^{6}$

Im Dialogus kommen neben den Remedia amoris vorwiegend Allusionen auf die Metamorphoses vor, außerdem auf alle anderen Werke des aus Sulmo stammenden Dichters; sogar gibt es einen Passus, der auf eine pseudo-ovidianische Elegie (De pulice) anspielt. Dieser Abschnitt, in dem es um einen Floh geht, der zwischen den Schenkeln der geliebten Frau horstet, wurde von den Zeitgenossen Trösters mit Vorliebe zitiert.

Tu laceras corpus tenerum, durissime, morsu; cuius quum fuerit plena cruore cutis

(...)

Ah! piget, et dicam, quum strata puella recumbit,

tu femur avellis, cruraque aperta subis.

(Ps.-Ovid. Pulex 3-4 und 11-12)

Cur iam te aut pulicem inter dulcissimae tuae amicae crura succi pleni recubantem (...) esse optas...8

Das damals noch Ovid zugeschriebene Werk, das nach dem heutigem Stand von Ofilius Segrianus verfasst wurde, trifft außerdem nicht nur in der Novelle Historia de duobus amantibus des Piccolomini an, sondern kommt auch in einem Epigramm von Janus Pannonius vor, ${ }^{9}$ der ein gemeinsamer Freund von Tröster und Piccolomini war. Dieses Beispiel illustriert das gleiche Interesse und Meinung des im weiteren Sinne verstandenen Freundeskreises, sowie ihre gemeinsame Sprache, gemeinsame Quellennutzung, obwohl es keine Angabe über den eventuellen Ovid-Band Trösters gibt.

\footnotetext{
${ }^{6}$ RUPPRICH (Anm. 1) 189-190.

${ }^{7}$ Vgl. JanKOVITS, L.: Accessus ad Janum. A müértelmezés hagyományai Janus Pannonius költészetében [Die Traditionen der Interpretation in der Dichtkunst von Janus Pannonius]. Budapest 2002, 7981 , insbes. 80.

${ }^{8}$ RUPPRICH (Anm. 1) 192.

${ }^{9}$ Wie das schon von László Jankovits beweist wurde: JANKOVITS (Anm. 7) 79-81. Zu weiteren schlüpfrigen Flöhen s. SZENTMÁRTONI SZABÓ, G. - VIRÁGH, L.: Megzenésített magyar szitkozódás és a pozsonyi bolhák egy Lassus-motettában [Vertonte ungarische Schimpferei und die Preßburger Flöhe in einer Lassus-Motette]. Irodalomtörténeti Közlemények 105 (2001) 341-358.
} 


\section{SENECA}

In reichlichem Maße sind im Dialogus Auszüge aus den Tragödien von Seneca zu finden. Der Meister Trösters, Piccolomini zitierte aus der Octavia, als er Amor definieren wollte:

Vis magna mentis blandus atque animi calor

Amor est; iuventa gignitur, luxu otio

nutritur inter laeta Fortunae bona.

Quem si fovere atque alere desistas, cadit

brevique vires perdit extinctus suas.

(Sen. Oct. 562-566)

Amor, ut in tragediis inquit Seneca, nihil aliud est, nisi vis quaedam magna mentis, blandusque animi calor, qui iuventa gignitur, luxu, otio, et inter laeta fortunae bona nutritur.

(Eneas Silvius Piccolomini: De remedio amoris illiciti) ${ }^{10}$

Vis magna mentis (...) qui luxu otioque gignitur et inter laeta bona fortunae nutritur, et quem si alere desinas, brevis extinctus suas perdit vires. ${ }^{11}$

Diese Stelle gilt als richtiges Beispiel für die Zitiertechnik von Tröster: Er führt den gleichen Passus wie Piccolomini an, aber nicht von dem Meister, sondern von den Quellen selbst zitierend. Während Piccolomini offen auf seine Quellen verwies, erwähnte sie Tröster namentlich üblicherweise eigentlich nicht, dennoch zitierte er längere Abschnitte. Im Dialogus werden neben der Octavia die folgenden Tragödien wörtlich zitiert: Hercules furens, Phaedra, Oedipus und der Hercules Oetaeus, dessen Autor bis heute umstritten ist. Wenn wir diese Angaben mit den bekannten Stücken der Bibliothek von Tröster vergleichen, lässt sich Folgendes feststellen: Gegenwärtig wissen wir Bescheid nur über die Episteln von Seneca, dieses Exemplar ist jedoch nicht mehr verfügbar. Über einen Band mit den Tragödien ist keine Angabe vorhanden.

\section{TERENZ UND PLAUTUS}

Obwohl es im Titel des Werkes heißt, dass es ein Dialogus ist, war die Gattungseinordnung während des 15. Jahrhunderts nicht so eindeutig. In seiner Studie über die neulateinischen Dramen schrieb Rudolf Wolkan, dass man ,zur Zeit (...) schon Dialoge ohne irgendwelchen dramatischen Kern als Dramen" betrachtet hatte, und unter anderem erwähnte er den Dialogus von Tröster als Beispiel. Wolkan stellte fest, dass die dramatischen Momente aus diesen Werken fehlen: „Wie in Zeiten des Übergangs alle Grenzen unsicher werden, so auch hier die zwischen Dialog und Dra-

\footnotetext{
${ }^{10}$ WOLKAN (Anm. 5) 34-35.

${ }^{11}$ RUPPRICH (Anm. 1) 194.
} 
ma; wir haben also nur die Möglichkeit, die Werke der Humanisten, insofern sie an klassische Muster sich lehnen und als Dramen gelten wollen, zu überblicken und dürfen dabei nicht vergessen, daß selbst so prosaische Stilübungen wie die Colloquia (Gespräche) des Erasmus aufgeführt werden konnten.“'12

Der Dialogus ruft in vielen Hinsichten die Gattung der Komödie wach. Das zeigen v.a. die durchgehend auftauchenden situationskomischen Elemente, die mit den Personen und den sprechenden Namen, die die römische Gattung atellana hervorrufen, in Zusammenhang stehen. Die zwei Hauptfiguren bekamen sprechende Namen: der Adressierte des Dialogus, Wolfgang Forchtenauer kommt als Philostratus (d.h. ,von Liebe erschüttert') vor, während Tröster selbst die Rolle seines Freundes und Gesprächspartners, Eudion (d.h. ,guter ratender ${ }^{6}$ ) spielt. Philostratus leidet an der Liebeskrankheit und Eudion, der die Maske eines Arztes trägt, hilft ihm bei der Heilung. Das Gejammer und Selbstmitleid des Philostratus wirken dem Verhalten des Pappus der Gattung atellana ähnlich, während Eudion dem viel plappernden und durchgehend hungrigen Bucco gleicht. Auf diese Weise stellt sich Eudion-Tröster, der Autor selbst als Parasit vor: Diese Rolle ist eine mögliche Bestimmung des Dichterwesens in der Sprache der römischen Komödie. Denn „der Mäzen (und/oder der Staat) alimentiert den Dichter, der aus dem Verkauf seiner Wörter und Sprache lebt, und vorwiegend wenn er Komödien schreibt, ist seine Ware wie das Gut des Parasiten. Ihre sozialen Positionen ähneln sich auch: Der Dichter ist ein Klient, wirklichrechtlich, doch symbolisch auch; sein sprechende Zunge ist Sklave seiner speisenden Zunge." ${ }^{\prime 13}$

Neben den Obigen verstärken die Terenz- und Plautus-Zitate den komischen Charakter:

Quem ego credo manibus pedibusque obnixe omnia facturum, magis id adeo mihi ut commodet. (Ter. And.161-162)

Scio, manibus pedibusque omnia obnixe facturum, mihi ut commodet. ${ }^{14}$

Aut qua quaerere insistam via?

(Ter. Phor. 192)

Quem ubi queram? Aut qua insistam via? ${ }^{15}$

Die Geschichte der frühneuzeitlichen Komödie nahm seinen Anfang mit zwei, kurz auf einander folgenden Ereignissen: Einerseits fand Nicolaus Cusanus 1429 die Plautus-Komödien, die bis dahin als verloren galten, andererseits entdeckte Giovanni Aurispa 1433 den Terenz-Kommentar von Donatus. Aus der Bibliothek Trösters ken-

${ }^{12}$ WOLKan, R.: Das neulateinische Drama. In ARnOlD, R. F. (Hrsg.): Das deutsche Drama. München $1925,125-126$.

${ }^{13}$ DÉR, K.: „Dagadt has - derüs szív“ (A foglyok 771). Lakomák és nagyevők Plautus színpadán [Konvivien und Fresser auf der Bühne von Plautus]. Okor 4 (2005/3) 25. Vgl. DAmON, C.: The Mask of the Parasite. A Pathology of Roman Patronage. Michigan 1997. Im Zitat wird Zunge als lingua, d.h. ,Zunge' sowie ,Sprache' verstanden.

${ }_{15}^{14}$ RUPPRICH (Anm. 1) 186.

${ }^{15}$ RUPPRICH (Anm. 1) 186 
nen wir heute einen Plautus-Band, der aber nur die acht Komödien enthält, die früher auch bekannt waren; ${ }^{16}$ nach der eingehenden Untersuchung des Dialogus scheint Tröster die neu entdeckten Komödien entweder gar nicht gelesen zu haben, oder er kannte sie nicht so tiefgründig, dass er aus ihnen zitiert hätte. Keine der erwähnten Entdeckungen scheint eine Wirkung auf den Dialogus Trösters ausgeübt zu haben. Wir haben Angaben weder über einen eigenen Terenz-Band, noch über einen eigenen Kommentar von Terenz, obwohl der Dialogus von Terenz-Zitaten wimmelt. Dass Terenz einer seiner Lieblingsautoren war, beweist ein Hinweis im Dialogus: Als EudionTröster seine Schuld bezahlen sollte, versuchte er das eher metaphorisch zu tun: Sed suppleat Terentius Ciceroque meus tuam mihi creditam pecuniam. ${ }^{17}$

Neben den inhaltlichen Kontexten lohnt es sich anzumerken, dass die Abschriften des Werkes teilweise in Komödiensammlungen aufbewahrt wurden, und ein reichlich mit Notizen versehenes Exemplar enthält sogar dramaturgische Hinweise. ${ }^{18}$ Das legt die Schlussfolgerung nahe, dass der Dialogus wenn auch nicht aufgeführt, so zumindest als Lesedrama, Bühnenwerk (auch) gelesen wurde.

\section{VERGIL UND DIE AENEIS}

Tröster zitierte alle drei wohlbekannten Werke von Vergil gerne. ${ }^{19}$ Selbstverständlich bietet die Aeneis, die in der Schule Lesestück war, die meisten Allusionen, aber man kann auch von den Eclogae, die ebenfalls humanistischer Vorlesungstext waren, Zitate finden, und erstaunlicherweise stammen zahlreiche Textstellen aus den Georgica. Aus dem Letzteren führt Tröster bei der Beschreibung der Symptome des liebenden Mannes typischerweise den Abschnitt an, in dem der sterbende Hengst seine Seele verhaucht. ${ }^{20}$

Die Allusion auf die Aeneis scheint einer besonderen Beachtung würdig zu sein. Im Prolog verweist der Autor auf den Passus über die Tore des Schlafs, der offenbar eine der umstrittensten und fragwürdigsten Stellen des Epos ist. In der Aeneis lautet der Passus so:

Sunt geminae Somni portae, quarum altera fertur cornea, qua veris facilis datur exitus umbris, altera candenti perfecta nitens elephanto, sed falsa ad caelum mittunt insomnia Manes. (Aen. VI 893-896)

Vor einigen Jahren verfasste Máté Vince einen tiefgründigen und umfassenden Aufsatz über die Interpretation der Tore des Schlafs, im Folgenden werde ich mich an

\footnotetext{
${ }^{16}$ Lehmann (Anm. 3) 658. Das Exemplar s. München, UB, cod. ms. $2^{\circ} 545$.

${ }^{17}$ RUPPRICH (Anm. 1) 187

${ }^{18}$ St. Pölten, Diözesanarchiv, Hs 63, 155r-164v.

${ }^{19}$ Über einen eigenen Vergil-Band ist keine Angabe vorhanden.

${ }^{20}$ Georg. III 500-508, vgl. RUPPRICH (Anm. 1) 187.
} 
seine Ergebnisse anlehnen. ${ }^{21}$ Nachdem er von Anchises das Auspizium über die $\mathrm{Zu}$ kunft des römischen Volks erhielt, verließ Aeneas die Unterwelt durch das Tor des falschen Schlafs, d.h. durch das Elfenbeintor. Die Interpretation dieser Stelle beunruhigte schon den antiken Kommentator Servius, und seitdem sind zahlreiche interessante Erklärungen erschienen, wie man die Stelle besser verstehen könnte. Ihre Interpretation wurde trotzdem manchmal noch unklarer. Die am meisten philologische Lösung stammt womöglich von Eduard Norden, dessen Meinung nach sich die Tore des Schlafs um Mitternacht schließen, weswegen Aeneas durch das Elfenbeintor abtreten musste. ${ }^{22}$ Alle Kommentatoren sind aber damit einverstanden, dass die Interpretation der Szene sich auf die Interpretation des ganzen Epos auswirkt, deswegen können wir diese Stelle nicht außer Acht lassen. Tröster schreibt im Prolog an seinen Freund Wolfgang Forchtenauer:

Si quid aut impudicum aut minus honestum dixisse aut fecisse te dicam, id non in re esse, sed finxisse me puta, qut nihil credas, somnium tamen putabis, et per corneam, minime per eburneam, exisse portam, tu te iudicabis. ${ }^{23}$

Aus dem Briefwechsel von Tröster, der teilweise erhalten ist, stellt sich heraus, dass er ein früheres Konzept des Dialogus an seine Meister und Freunde Johannes Hinderbach und Eneas Silvius Piccolomini geschickt hatte, damit sie den Text korrigieren und Kritik darüber geben. Piccolomini ging auf diese Stelle in seinem Antwortbrief ein:

Cum de porta cornea efficis mentionem, si futile somnum tuum, nihil veri habere intellegis. Non recte locutus es, veris enim hac porta datur exitus umbris, ut Maroni placet.

$$
\text { (Epistola ad Johannem Tröster) }^{24}
$$

Piccolomini setzte aus, dass Tröster die Aeneis-Stelle missverstanden hatte, und als er auf das fiktive Wesen des Werkes verweisen wollte, habe er versehentlich dasjenige Tor erwähnt, durch welches die richtigen Träume die Unterwelt verlassen. Von den Varianten der erhaltenen Abschriften kann man nicht entscheiden, ob sie vor oder nach den Korrektur von Piccolomini entstanden, allerdings wurde die oben genannte Stelle in keiner der neun Handschriften ,verbessert“. Der andere Freund, Johannes Hinderbach meinte im Antwortbrief, dass die Wahl Forchtenauers für die Rolle des Philostratus unbegründet ist, denn offenkundig schaudert dieser vor der Laszivität (lascivia multum horrere). ${ }^{25}$ Jedoch ist Forchtenauer-Philostratus im Dialogus ein großer Frauenliebhaber: Wir lernen vier Damen kennen, in die er unlängst ungeheuer

${ }^{21}$ VINCE, M.: Ha az álom kapuja kitárul [Wenn sich das Tor des Schlafs öffnet]. In FERENCZI, A. (Hrsg.): A rejtélyes Aeneis [Die geheimnisvolle Aeneis]. Budapest 2005, 89-117.

${ }_{22}^{22}$ NORDEN, E.: P. Vergilius Maro: Aeneis Buch VI. Stuttgart-Berlin 1916, 348.

${ }^{23}$ RUPPRICH (Anm. 1) 182

${ }^{24}$ Aeneas Silvius Piccolomini: Opera quae extant omnia. Basel 1551, 951.

${ }^{25}$ PicCOLOMini (Anm. 24) 951. 
verliebt war. Die Lage wird dadurch verwickelter, dass einige unanständige Gedichte von Johannes Baptista Domisius Dionysii erhalten sind, die auch an Forchtenauer adressiert wurden. ${ }^{26}$ Die Lösung besteht vielleicht darin, dass Tröster die Aeneis-Stelle richtig verstand, und deswegen auf das Horntor bestand.

Máté Vince hebt den Aufsatz von Agnes Kirsopp Michels hervor: Michels erklärt das in der Szene erscheinende insomnium mit der Hilfe von Macrobius ${ }^{27}$ als solchen Schlaf/Traum, der ,in verwirrtem Nervenzustand auftritt, und deswegen keine verwendbare Bedeutung hat, man kann es nicht interpretieren, und nach seinem Ende wird er auch entfallen“. Andererseits ist das Wort falsa ihrer Meinung nach nicht als , lügend", sondern als ,unwahr" zu verstehen, und nach dem Erwachen wird dieser Schlaf/Traum keine Wirkung auf den Träumer haben. ${ }^{28}$ Aeneas scheint in den späteren Szenen des Epos, besonders im letzten nicht auf die stygischen Ratschläge seines Vaters zu hören, und er wird nicht von der pietas, sondern vielmehr vom furor geleitet. Forchtenauer soll sich aber auf seinen wahren Schlaf erinnern, obwohl er den Traum/Schlaf auch für Fiktion halten könnte, so dass die Anspielung auf das Horntor sein scheinheiliges Verhalten entlarvt. Wenn wir die Wörter Hinderbachs als ironische Bemerkung interpretieren, gibt es keinen Widerspruch mehr: Niemand kann unberührt sein, dem seine Freunde über Liebesheilmittel, Freundinnen, Zahne habende cunnus (si dentigerum os auidus uel retia cunnus / ferret) schreiben: Es ist möglich, dass unter den Freunden eben Forchtenauer am mutwilligsten war. Die im Prolog stehende Aeneis-Allusion gilt folglich als die Entlarvung der Laszivität von Forchtenauer und das Augenzwinkern der Freunde, während die Ehre des Adressierten nicht beschmutzt wird.

\section{VALERIUS MAXIMUS, PLUTARCH ODER AVICENNA?}

Abschließend bleibt eine vermutlich nicht zu beantwortende Frage. Philostratus offenbart weder seine Liebeskrankheit, noch den Gegenstand seiner Liebe, deswegen muss Eudion diese auf folgende Weise herausfinden: Er nimmt das Handgelenk des Philostratus und beschreibt verschiedene Frauen, während er seinen Puls misst. Als sich der Puls verstärkt, das Herz durcheinander schlägt, und Philostratus erbleicht, ist die gesuchte Person gefunden. In der Szene können die Beherrscher der antiken Literatur den Fall des Antiochus gleich erkennen, der sich in seine Stiefmutter verliebte und sein Arzt die Krankheit anhand der folgenden Merkmale identifizierte: Antiochus errötete und sein Atem wurde schneller, wenn Stratonica erschien, und er erbleichte,

\footnotetext{
${ }^{26}$ München, BSB, clm 418, fol. 129v-131r und clm 651, fol. 4r-6r. Auf diese Gedichte machte mich Herr Franz Fuchs aufmerksam, der mir auch die Kopie der Handschriften zur Verfügung stellte, ich möchte ihm hiermit dafür danken. Es würde sich lohnen, diese Gedichte mit dem Epigramm von Janus Pannonius über die vulva von Ursula zu vergleichen, s. ep. 197. Vgl.: MAYER, J. - TÖRÖK, L. (Eds.): Iani Pannonii opera quae manserunt omnia. Vol. I. Epigrammata, Fasc. I. Textus. Budapest 2006, 145-146.

${ }^{27}$ Macr. Comm. in Somn. Scipionis I 3. 2-6.

${ }^{28}$ VinCE (Anm. 21) 112. Vgl. MicheLS, A. K.: The Insomnium of Aeneas. Classical Quarterly N. S. 31 (1981) 144-145.
}

Acta Ant. Hung. 54, 2014 
wenn sie abtrat; während sein Puls mal schneller, mal langsamer wurde. ${ }^{29}$ Die Geschichte wurde nicht nur von Valerius Maximus, sondern vorher von Plutarch auf Altgriechisch, ${ }^{30}$ dann von Appian ${ }^{31}$ und Pseudo-Lukian ${ }^{32}$ auch verewigt. ${ }^{33}$

Woher kannte aber Tröster die Pulsdiagnostik? Er erwähnte Valerius Maximus, dessen Facta et dicta memorabilia im Spätmittelalter und in der Frühen Neuzeit allgemein bekannt und populär war, gar nicht, als er seine Lieblingsautoren aufführt: ${ }^{34}$

Aut quorsum me defatigem, in Parnassum nisi, ibi [sc. Eudion] in arce Tarpeia aut cum Cordubensibus Lucano Senecaque, aut Paeligno Nasone, aut Arpinate illo Cicerone, vel Patavino illo Livio, aut Maeonio illo Homero, aut Mantuano illo Virgilio certat. ${ }^{35}$

Ebenfalls ist kein Exemplar aus der Rekonstruktion seiner Bibliothek bekannt. Er erwähnte weder Plutarch, noch Appian, ${ }^{36}$ deren Werke er in lateinischer Übersetzung wohl kannte, obendrauf verschaffte er sich eine Handschrift der Vitae parallelae zufälligerweise 1454, im Jahr der Verfassung des Dialogus. ${ }^{37}$ Von den Kennern der Pulsdiagnostik benannte er dagegen den berühmten arabischen Arzt Avicenna, der eine umfassende Bildung in der griechischen Literatur besaß. In seinem Werk Canon medicinae bespricht Avicenna die Liebeskrankheit, wie er sagt: iliscii unter den melancholischen Krankheiten und diagnostiziert sie nach den gleichen Symptomen. Es ist fraglich, inwiefern die Texte des Plutarchs oder des Appians sich in den arabischen Gebieten verbreiteten, d.h. ob Avicenna die Geschichte der Liebe des Antiochus kannte. Offenkundig wurden die Lehren des Aristoteles und Platons durch die Tätigkeiten der Kommentatoren der peripatetischen und neuplatonischen Schule in der arabischen Welt schon während des 10. Jahrhunderts verbreitet, und Avicenna selbst kommentierte die Übersetzung der Poetik. So ist es nicht auszuschließen, dass Tröster bei der Beschreibung der Anleitungen der Pulsdiagnostik eigentlich eine durch die Araber vermittelte antike griechische Quelle zitierte, wohl unbewusst.

Die Reihe ließe sich noch lange fortsetzen: Tröster war nicht nur in den Werken antiker Autoren bewandert, sondern er zitierte die mittelalterlichen und frühneuzeitlichen Schriftsteller auch gerne. In diesem Beitrag konnte aber keine Vollständigkeit angestrebt werden. Über die Bildung und die literarische Tätigkeit des vergesse-

${ }^{29}$ Val. Max. Facta et dicta memorabilia 5. 7. ext. 1.

${ }^{30}$ Plut. Dem. 38.

${ }^{31}$ App. Hist. Rom. XI. (De rebus Syriacis) 59-61.

${ }^{32}$ Luk. De dea Syria 17-18.

${ }^{33}$ Vgl. STeChOW, W.: The Love of Antiochus with Faire Stratonica. Art Bulletin 27 (1945) 221237.

${ }^{34}$ Allem Anschein nach galten Seneca und Lukan als Lieblingsautoren auch für Johannes Vitéz de Zredna, vgl. den Aufsatz über seine antiken Vorbilder: BORONKAI, I.: Vitéz János és az ókori klasszikusok [Johannes Vitéz de Zredna und die antiken Klassiker]. KARDOS, T. - V. KovÁCS, S. (Hrsg.): Janus Pannonius. Tanulmányok. Budapest 1975, 219-232.

${ }^{35}$ RUPPRICH (Anm. 1) 186. $44 \mathrm{a}$ in $2^{\circ}$

${ }^{36}$ Sein eigenes Exemplar enthält die Übersetzung von Petrus Candidus, s. München, UB, A. graec.

${ }^{37}$ Von mehreren übersetzt, s. München, UB, Cod. 549. Vgl. CORTESI (Anm. 4) 227. 
nen Autors wird die kritische Edition ein differenzierteres Bild liefern. Auf diese Weise wird nicht nur der ungarische Humanismus weiter erforscht, sondern es wird mit plastischen Beispielen illustriert, welche Techniken (aemulatio, imitatio) ein Frühhumanist benutzte, der den Parnass besteigen wollte.

\author{
Emőke Rita Szilágyi \\ Institut für Literaturwissenschaft, Abt. Renaissance \\ Ungarische Akademie der Wissenschaften \\ Ménesi út 11-13. \\ 1118 Budapest \\ Ungarn \\ szilagyi.emoke.rita@btk.mta.hu
}

